\title{
Regulators of Rho GTPases in Neuronal Development
}

\author{
Mitsuko Watabe-Uchida, ${ }^{1}$ Eve-Ellen Govek, ${ }^{2}$ and Linda Van Aelst ${ }^{1}$ \\ ${ }^{1}$ Cold Spring Harbor Laboratory, Cold Spring Harbor, New York 11724, and ${ }^{2}$ The Rockefeller University, New York, New York 10021
}

\begin{abstract}
The formation and elaboration of axonal and dendritic morphologies are fundamental aspects of neuronal polarization critical for information processing. In general, developing CNS neurons elaborate one axon and multiple dendrites in response to intracellular and extracellular cues, so as to transmit and receive information, respectively. The molecular mechanisms underlying axon-dendrite polarity are complex and involve the integration of numerous signaling pathways that impinge on the cytoskeleton. One group of proteins, the Rho GTPases, has emerged as key integrators of environmental cues to regulate the underlying axonal and dendritic cytoskeletons. Here, we discuss the role of regulators of the Racl GTPase in axon development and highlight the importance of both actin and microtubule remodeling in this process.
\end{abstract}

Key words: neuronal polarity; axon formation; Rho GTPases; cytoskeleton; DOCK7; Tiam-1

During development, the majority of CNS neurons polarize to elaborate one long, slender axon and multiple shorter, tapering dendrites. Communication between neurons involves the formation of synapses between axons of presynaptic neurons and dendrites of postsynaptic neurons. Generally, axons send signals, and dendrites receive signals. Thus, both axonal and dendritic morphologies, which are primarily determined by their underlying cytoskeletons, will have an important impact on the processing of neuronal information. Multiple environmental cues, including neuronal activity, the neurotrophin family of growth factors, and extracellular guidance molecules, influence the development, patterning, size, and shape of dendrites and axons (Guan and Rao, 2003; Jan and Jan, 2003; Van Aelst and Cline, 2004; Wiggin et al., 2005). Defining how neurons acquire their polarity, size, and shape during development and how learning and experience influence morphological changes that alter the functional connectivity between presynaptic and postsynaptic cells has been a major challenge. One group of signaling molecules, the Rho GTPases, have emerged as key integrators of environmental cues to regulate the underlying axonal and dendritic cytoskeletons (Van Aelst and Cline, 2004) (see also below).

As mentioned above, the formation and elaboration of axonal and dendritic morphologies are fundamental aspects of neuronal polarization critical for information processing. In developing hippocampal and cortical neurons, multipolar cells extending several minor neurites are observed as an intermediate stage before axonal extension occurs (Goslin and Banker, 1989; Noctor et al., 2004; Nakahira and Yuasa, 2005). Typically, one of these neurites rapidly extends to form the axon, and several days later, the remaining neurites will develop into dendrites. The first sign of

\footnotetext{
Received Sept. 18, 2006; accepted Sept. 18, 2006.

This work was supported by grants from the National Science Foundation and the National Alliance for Autism Research.

Correspondence should be addressed to Linda Van Aelst, Cold Spring Harbor Laboratory, 1 Bungtown Road, Cold Spring Harbor, NY 11724. E-mail: vanaelst@cshl.edu.

DOI:10.1523/JNEUROSCI.4084-06.2006

Copyright $\odot 2006$ Society for Neuroscience $\quad$ 0270-6474/06/2610633-03\$15.00/0
}

an axon is generally hailed as evidence of morphological polarization. The molecular and cellular mechanisms that underlie axon-dendrite polarity are complex. They involve the integration of cues and signals that define which protrusion/neurite is to become the axon and those that promote rapid elongation of the nascent axon and sustain axonal outgrowth. A number of models have been proposed on how the axon is specified, including centrosome positioning in postmitotic neurons and the generation of a positive feedback loop (Zmuda and Rivas, 1998; Shi et al., 2003; de Anda et al., 2005; Ye and Jan, 2006). Preferential and persistent accumulation or activation of the growth-promoting machinery in just one neurite, or at a particular location on the cell body, of an unpolarized neuron is likely to be key in the fast elongation of the future axon (Jiang and Rao, 2005; Jacobson et al., 2006). Intracellular mechanisms that enhance neurite/axon growth involve changes in both actin and microtubule (MT) networks. For example, locally destabilizing actin filaments can induce axon formation, and actin instability appears to be highest in the neurite destined to become the axon (Bradke and Dotti, 1999; Da Silva et al., 2005). There is also a dramatic influx of MTs into the nascent axon that together with enhanced MT growth is believed to contribute to neurite/axon elongation ( $\mathrm{Yu}$ and Baas, 1994; Yu et al., 2001; Baas, 2002).

A number of signaling molecules that have been linked either directly or indirectly to the regulation of the cytoskeleton have been implicated in different steps of axon-dendrite polarization. These molecules include phosphatidylinositol 3-kinase and its product PIP3, the Akt/GSK-3 $\beta /$ CRMP-2 or adenomatous polyposis coli complex, the evolutionarily conserved Par3/Par6/aPKC complex, SAD (synapses of the amphid defective) kinases, Ras and Rho family members, and the membrane enzyme PMGS, which influences the activities of Rac and Rho GTPases (for review, see Arimura and Kaibuchi, 2005; Govek et al., 2005; Kishi et al., 2005; Wiggin et al., 2005) (see also second and third section of this Mini-Review series).

Members of the Rho family of GTPases, including Rac, Cdc42, and RhoA, function as binary molecular switches by cycling be- 
tween an active GTP-bound state and an inactive GDP-bound state. Their activity is determined by the ratio of GTP to GDP in the cell and can be influenced by a number of different regulatory molecules (Van Aelst and D'Souza-Schorey, 1997). These include positive regulators, guanine-nucleotide exchange factors (GEFs), and negative regulators, GTPase activating proteins (GAPs), and guanine nucleotide dissociation inhibitors (Hoffman et al., 2000; Schmidt and Hall, 2002; Bernards, 2003). Only GTP-bound Rho GTPases can interact with effector proteins, which in turn mediate their biological activities. RhoA, Rac1, and Cdc42 are best known for their characteristic effects on the actin cytoskeleton but more recently have also been shown to influence MT organization (Van Aelst and D'Souza-Schorey, 1997; EtienneManneville and Hall, 2001; Gundersen et al., 2004). As key modulators of the cytoskeleton, these proteins have been found to play important roles in various aspects of neuronal development, including neuronal migration, neurite/axon formation and outgrowth, as well as dendrite and dendritic spine formation and maintenance (Govek et al., 2005). The importance of Rho signaling in neuronal development is also highlighted by the findings that mutations in their regulators and effectors are found to underlie or contribute to various neurological disorders (Newey et al., 2005). How could these numerous varied developmental processes all require Rho GTPases? Specificity may be conferred through spatio-temporal regulation of the GTPase activity by the distinct GEFs and GAPs. Indeed, GEFs have been proposed to contribute to signaling specificity by associating with scaffolding molecules that link them and the GTPase to specific effectors (Buchsbaum et al., 2002, 2003; Jaffe et al., 2004). Here, we will mainly discuss the role of regulators of the Rac1 GTPase in axondendrite polarity.

Several studies indicate that localized activation of the Rac1 GTPase contributes to the rapid elongation of the nascent axon (Kunda et al., 2001; Ng et al., 2002; Chuang et al., 2005; Da Silva et al., 2005; Nishimura et al., 2005). Insights into the molecular regulation of Racl GTPase activity important for this process have recently been obtained. The Rac-specific GEFs, Tiam-1 (invasion-inducing T-lymphoma and metastasis 1) and a Tiam1-related protein, STEF, have been reported to regulate Rac1driven actin remodeling to promote elongation of the future axon. A study by Kunda et al. (2001) suggests that Tiam-1 affects neuronal polarization through actin reorganization in the tip of axonal growth cones of rat hippocampal neurons. Overexpression of Tiam-1 promotes the formation of several long, thin axon-like processes, whereas suppression of Tiam-1 prevents axon formation. Kaibuchi and colleagues (Nishimura et al., 2005) found that STEF (which also induces multiple axon-like neurites) is present in a complex with Par-3, Par-6, aPKC, and GTP-bound Cdc42 and that STEF directly interacts with Par3. They also demonstrated that interfering with Par3 function inhibited Cdc42-induced Racl activation in N1E-115 neuroblastoma cells (Nishimura et al., 2005). Because Cdc42 can interact with Par-6 in neurons (Schwamborn and Puschel, 2004), a model was proposed in which Cdc42 signals to STEF/Tiam-1 through the Par-3/Par-6/aPKC complex to activate Rac and trigger actin remodeling and neurite/axon elongation (see second section of this Mini-Review series).

The above studies indicate that activation of a Rac signaling pathway that controls actin remodeling contributes to the development of the axon. Interestingly, recent work by our group has delineated a distinct pathway in which local activation of a novel Rac-specific GEF, DOCK7, regulates Rac activity to inactivate the MT destabilizing protein stathmin/Op18 and promote axon for-

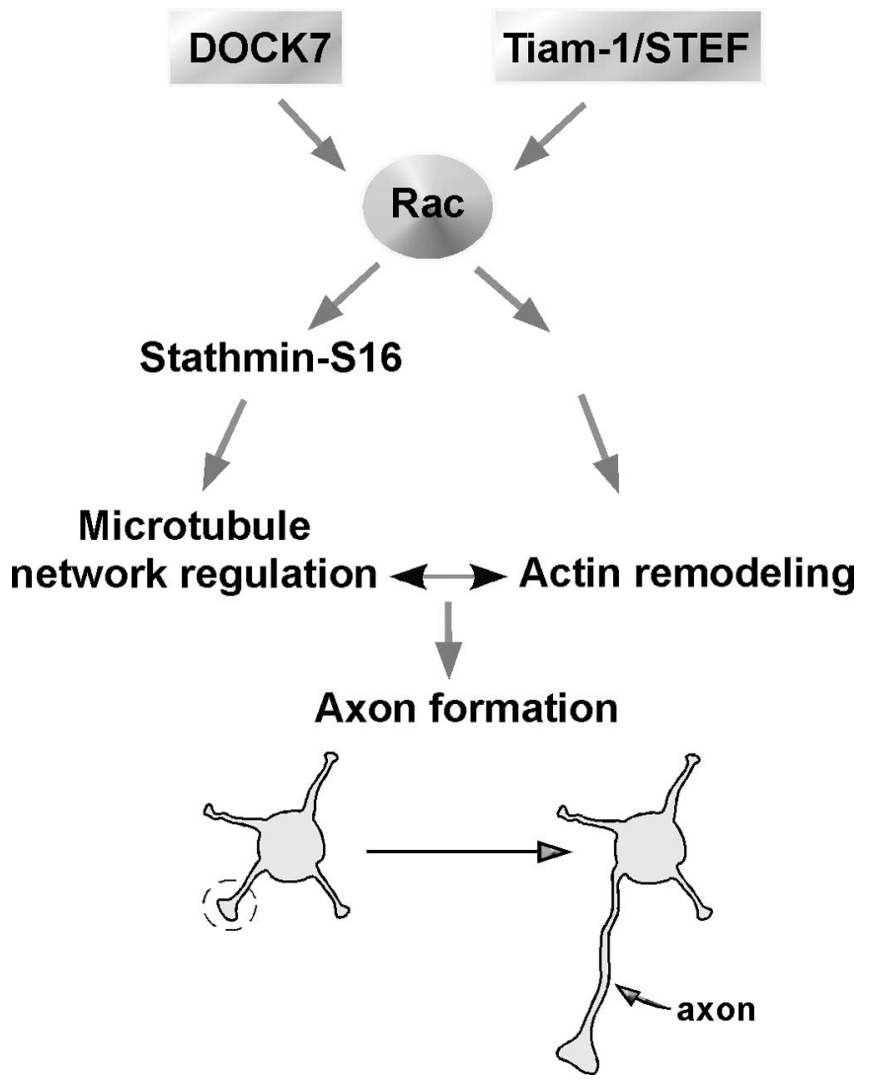

Figure 1. Regulators of Rac1 GTPase in axon development of hippocampal neurons. DOCK7 and Tiam-1/STEF trigger the activation of Rac1 GTPase to regulate MT and actin remodeling, respectively. Both events are important for the rapid elongation of the neurite to become the axon (see text for more details).

mation (Watabe-Uchida et al., 2006). We identified DOCK7 in a screen for upstream regulators of the Rac1 GTPase. DOCK7 is a member of the evolutionarily conserved DOCK180-related protein superfamily, which emerged as a new class of activators of the Rho GTPases. The DOCK7 protein is highly expressed in the developing brain, and significantly it is asymmetrically distributed in unpolarized stage 2 hippocampal neurons and selectively expressed in the axon. Knockdown of DOCK7 expression prevents axon formation, whereas overexpression induces the formation of multiple axons. Interestingly, we obtained evidence showing that DOCK7 and subsequent Rac activation lead to phosphorylation (at serine 16) and inactivation of the MT destabilizing protein stathmin/Op18 in the nascent axon and that this event is important for axon development (Watabe-Uchida et al., 2006). In particular, the levels of phosphorylated stathmin-S16 are higher in the nearly formed axon than in the future dendrites, and expression of a phosphorylation-deficient stathmin mutant (stathmin-S16A) perturbs axon formation and suppresses the DOCK7-induced formation of multiple axons. Thus, our study links the Rac activator DOCK7 to a MT regulatory protein and supports the idea that spatially controlled regulation of the MT network is important for axon formation and neuronal polarization. How DOCK7 becomes concentrated in just one neurite of stage 2 neurons and is selectively expressed in the axon is currently unknown.

An intriguing question is why more than one activator (e.g., DOCK7 and STEF/Tiam-1) is required for Rac in the developing axon? Genetic studies in Drosophila have elegantly illustrated that distinct Rac-GEFs couple to different downstream effector path- 
ways and that each of them contributes to axonal outgrowth $(\mathrm{Ng}$ and Luo, 2004). Therefore, it is tempting to speculate that spatial and temporal activation of DOCK7 and STEF/Tiam-1 engages different effector pathways that contribute to the MT and actin reorganization needed for axon formation during polarized growth (Fig. 1). Whether different extracellular cues are involved in the activation of DOCK7 and STEF/Tiam-1 remains to be seen. Presumably, there will be cross talk between these pathways, particularly because the actin and MT cytoskeletal systems are known to feedback on each other (Waterman-Storer et al., 1999; Rodriguez et al., 2003). This may potentially contribute to the local increase in Tiam-1/STEF and/or DOCK7 activities.

Clearly, the establishment of axon-dendrite polarity is a complex process that involves numerous molecular players besides the Rho GTPases. In the future, it will be important to determine how all of these signaling pathways and mechanisms are integrated to mediate the development and growth of the axon and dendrites. In addition, studies involving real-time imaging of cytoskeletal dynamics during axon formation will be required to further elucidate their contributions to this process in vivo.

\section{References}

Arimura N, Kaibuchi K (2005) Key regulators in neuronal polarity. Neuron 48:881-884.

Baas PW (2002) Neuronal polarity: microtubules strike back. Nat Cell Biol 4:E194-E195.

Bernards A (2003) GAPs galore! A survey of putative Ras superfamily GTPase activating proteins in man and Drosophila. Biochim Biophys Acta 1603:47-82.

Bradke F, Dotti CG (1999) The role of local actin instability in axon formation. Science 283:1931-1934.

Buchsbaum RJ, Connolly BA, Feig LA (2002) Interaction of Rac exchange factors Tiam1 and Ras-GRF1 with a scaffold for the p38 mitogenactivated protein kinase cascade. Mol Cell Biol 22:4073-4085.

Buchsbaum RJ, Connolly BA, Feig LA (2003) Regulation of p70 S6 kinase by complex formation between the Rac guanine nucleotide exchange factor (Rac-GEF) Tiaml and the scaffold spinophilin. J Biol Chem 278:18833-18841.

Chuang JZ, Yeh TY, Bollati F, Conde C, Canavosio F, Caceres A, Sung CH (2005) The dynein light chain Tctex-1 has a dynein-independent role in actin remodeling during neurite outgrowth. Dev Cell 9:75-86.

Da Silva JS, Hasegawa T, Miyagi T, Dotti CG, Abad-Rodriguez J (2005) Asymmetric membrane ganglioside sialidase activity specifies axonal fate. Nat Neurosci 8:606-615.

de Anda FC, Pollarolo G, Da Silva JS, Camoletto PG, Feiguin F, Dotti CG (2005) Centrosome localization determines neuronal polarity. Nature 436:704-708

Etienne-Manneville S, Hall A (2001) Integrin-mediated activation of cdc42 controls cell polarity in migrating astrocytes through PKCzeta. Cell 106:489-498.

Goslin K, Banker G (1989) Experimental observations on the development of polarity by hippocampal neurons in culture. J Cell Biol 108:1507-1516.

Govek EE, Newey SE, Van Aelst L (2005) The role of the Rho GTPases in neuronal development. Genes Dev 19:1-49.

Guan KL, Rao Y (2003) Signalling mechanisms mediating neuronal responses to guidance cues. Nat Rev Neurosci 4:941-956.

Gundersen GG, Gomes ER, Wen Y (2004) Cortical control of microtubule stability and polarization. Curr Opin Cell Biol 16:106-112.

Hoffman GR, Nassar N, Cerione RA (2000) Structure of the Rho family GTP-binding protein cdc42 in complex with the multifunctional regulator RhoGDI. Cell 100:345-356.
Jacobson C, Schnapp B, Banker GA (2006) A change in the selective translocation of the Kinesin-1 motor domain marks the initial specification of the axon. Neuron 49:797-804.

Jaffe AB, Aspenstrom P, Hall A (2004) Human CNK1 acts as a scaffold protein, linking Rho and Ras signal transduction pathways. Mol Cell Biol 24:1736-1746.

Jan YN, Jan LY (2003) The control of dendrite development. Neuron 40:229-242.

Jiang H, Rao Y (2005) Axon formation: fate versus growth. Nat Neurosci 8:544-546.

Kishi M, Pan YA, Crump JG, Sanes JR (2005) Mammalian SAD kinases are required for neuronal polarization. Science 307:929-932.

Kunda P, Paglini G, Quiroga S, Kosik K, Caceres A (2001) Evidence for the involvement of Tiam1 in axon formation. J Neurosci 21:2361-2372.

Nakahira E, Yuasa S (2005) Neuronal generation, migration, and differentiation in the mouse hippocampal primoridium as revealed by enhanced green fluorescent protein gene transfer by means of in utero electroporation. J Comp Neurol 483:329-340.

Newey SE, Velamoor V, Govek EE, Van Aelst L (2005) Rho GTPases, dendritic structure, and mental retardation. J Neurobiol 64:58-74.

Ng J, Luo L (2004) Rho GTPases regulate axon growth through convergent and divergent signaling pathways. Neuron 44:779-793.

Ng J, Nardine T, Harms M, Tzu J, Goldstein A, Sun Y, Dietzl G, Dickson BJ, Luo L (2002) Rac GTPases control axon growth, guidance and branching. Nature 416:442-447.

Nishimura T, Yamaguchi T, Kato K, Yoshizawa M, Nabeshima Y, Ohno S, Hoshino M, Kaibuchi K (2005) PAR-6-PAR-3 mediates Cdc42-induced Rac activation through the Rac GEFs STEF/Tiam1. Nat Cell Biol $7: 270-277$.

Noctor SC, Martinez-Cerdeno V, Ivic L, Kriegstein AR (2004) Cortical neurons arise in symmetric and asymmetric division zones and migrate through specific phases. Nat Neurosci 7:136-144.

Rodriguez OC, Schaefer AW, Mandato CA, Forscher P, Bement WM, Waterman-Storer CM (2003) Conserved microtubule-actin interactions in cell movement and morphogenesis. Nat Cell Biol 5:599-609.

Schmidt A, Hall A (2002) Guanine nucleotide exchange factors for Rho GTPases: turning on the switch. Genes Dev 16:1587-1609.

Schwamborn JC, Puschel AW (2004) The sequential activity of the GTPases Rap1B and Cdc42 determines neuronal polarity. Nat Neurosci 7:923-929.

Shi SH, Jan LY, Jan YN (2003) Hippocampal neuronal polarity specified by spatially localized mPar3/mPar6 and PI 3-kinase activity. Cell 112:63-75.

Van Aelst L, Cline HT (2004) Rho GTPases and activity-dependent dendrite development. Curr Opin Neurobiol 14:297-304.

Van Aelst L, D'Souza-Schorey C (1997) Rho GTPases and signaling networks. Genes Dev 11:2295-2322.

Watabe-Uchida M, John KA, Janas JA, Newey SA, Van Aelst L (2006) The Rac activator DOCK7 regulates neuronal polarity through local phosphorylation of stathmin/Op18. Neuron 51:727-739.

Waterman-Storer CM, Worthylake RA, Liu BP, Burridge K, Salmon ED (1999) Microtubule growth activates Rac1 to promote lamellipodial protrusion in fibroblasts. Nat Cell Biol 1:45-50.

Wiggin GR, Fawcett JP, Pawson T (2005) Polarity proteins in axon specification and synaptogenesis. Dev Cell 8:803-816.

Ye B, Jan YN (2006) Visualizing the breaking of symmetry. Dev Cell 10:411-412.

Yu W, Baas PW (1994) Changes in microtubule number and length during axon differentiation. J Neurosci 14:2818-2829.

Yu W, Ling C, Baas PW (2001) Microtubule reconfiguration during axogenesis. J Neurocytol 30:861-875.

Zmuda JF, Rivas RJ (1998) The Golgi apparatus and the centrosome are localized to the sites of newly emerging axons in cerebellar granule neurons in vitro. Cell Motil Cytoskeleton 41:18-38. 